\title{
Lightweight design of a shock tower based on topology and size optimization
}

\author{
Huajie Mao ${ }^{1.2 .3, ~ a, ~ W e n ~ F u ', b, ~ J i a n ~ L a n ~}{ }^{1.2 .3, c}$ and Xinhao Zhao ${ }^{1}$ \\ ${ }^{1}$ School of Materials Science and Engineering, Wuhan University of Technology, \\ Wuhan 430070, China; \\ ${ }^{2}$ Hubei Key Laboratory of Advanced Technology for Automotive Components \\ Wuhan University of Technology, Wuhan 430070, China; \\ ${ }^{3}$ Hubei Collaborative Innovation Center for Automotive Components Technology, \\ Wuhan University of Technology, Wuhan 430070, China \\ amaohj@whut.edu.cn, bfwflynn@163.com, cjlan@whut.edu.cn
}

\begin{abstract}
Keywords: Shock tower, Lightweight, Topology optimization, Size optimization, Aluminum alloy Abstract. The shock tower, serving as a critical component of the front car body, plays an important role in the stability and ride comfort of the vehicle. In this study, a lightweight optimal design was obtained based on the original steel structure. Stiffness requirements for optimization process were extracted from the original steel structure. Subsequently, topology optimization was implemented to gain the distribution of stiffeners, and size optimization was adopted to provide an optimal thickness for stiffeners. In addition, the choice of optimization space, which is critical and usually neglected, was taken into account. As a result, a detailed structure of the aluminum alloy shock tower for vacuum die casting was determined. Compared with the original steel structure, the weight of the final design is reduced by nearly $45 \%$ without sacrificing the functional performance.
\end{abstract}

\section{Introduction}

The shock tower is a critical component connecting the shock absorber and vehicle body. The vibration, caused by uneven road during the process of car driving, is attenuated by the shock absorber and then dispersed into the front vehicle body through the shock tower. The shock tower plays an important role in improving the ride comfort and $\mathrm{NVH}$ (noise, vibration and harshness) performance of the vehicle [1]. Traditional shock tower is welded by steel stampings with poor assembly accuracy and efficiency. Therefore, a structural optimization is needed.

Recently, aluminum alloy has received increasing utilization for the purpose of achieving automotive lightweight, while die-casting has been the main molding method. Changes in material processing performance can lead to the corresponding adjustment of the structure. Traditional parts design mainly start with the current practice based on standards and experiences. With the development of FEA (finite element analysis) technology, theory of optimization design [2-4] has been well established: Topology optimization is an effective method to obtain the optimal material distribution in the conceptual design stage, while size and shape optimization are used to adjust the local size and shape in the detailed design stage. Optimization methods should be combined with the specific structure of parts.

In this paper, topology optimization, based on SIMP method [5, 6], and size optimization were implemented on the front left shock tower with Altair Optistruct. An aluminum alloy shock tower for vacuum die casting was designed considering the overall performance of manufacturing feasibility, stiffness and environmental friendliness.

\section{Analysis of the original shock tower}

Assembly and structural model of the original shock tower. The original shock tower is a critical structure, connecting the shock absorber and front vehicle body, while bearing a variety of forces and moments. Fig. 1(a) shows the assembly of the front left car body. 


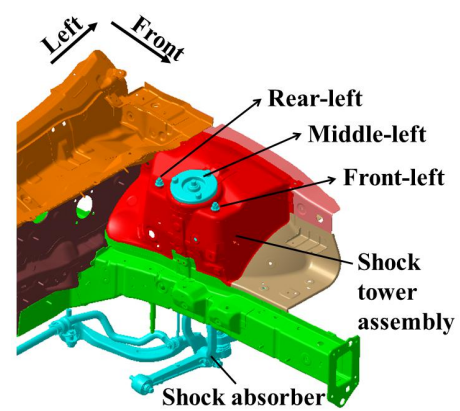

(a) Assembly of the front left vehicle body
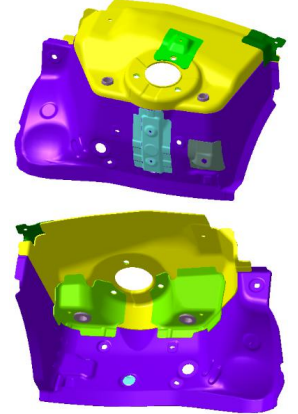

(b) detail structure of the shock tower

Fig.1 Assembly and structural model of the original shock tower

The original shock tower is composed of steel stampings with various thicknesses from $1.0 \mathrm{~mm}$ to $2.5 \mathrm{~mm}$. The detailed structure is expressed in Fig. 1(b). It includes several pieces of steel stampings, which reduce the assembly accuracy and efficiency of the shock tower as a whole. Furthermore, weld spots can result in stress concentration. Therefore, a lighter aluminum alloy vacuum die casting was designed to take place of the original steel structure, which can improve the fuel efficiency and assembly efficiency simultaneously.

Boundary condition of the original model. Multi-body dynamics analysis was performed to obtain the ultimate load of the shock tower under multi-loading system. There are 5 working conditions, and each condition contains three loading points which are displayed in Table 1.

Load and connection mode are displayed in Fig. 2. The weld spots between different stampings were modeled with hexahedron element to simulate the welding material. This area contact method (ACM) is more reasonable for it can avoid stress concentration. RBE2 elements were implemented on bolt holes and boundary welds which were fully constrained. The commercial software Altair Radioss was used to perform static analysis.

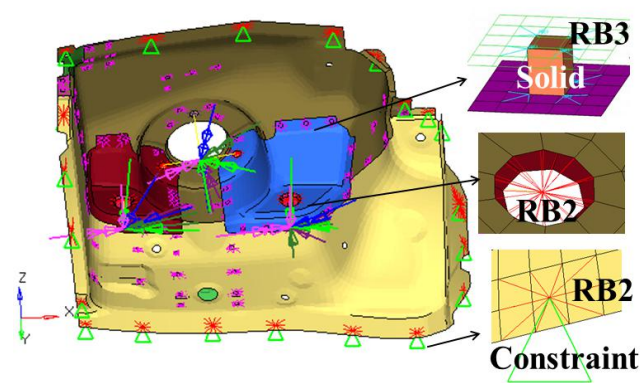

Fig. 2 Load and connection mode of the original shock tower

The result of static analysis. The structural stiffness $(\mathrm{k})$ can not be measured directly, It is correlated to the measured displacement (d) and force (F) by the Hooke law [7] :

$$
\mathrm{k}=F / d
$$

So the local stiffness can be expressed by nodal displacements. First-order eigenfrequency and displacements of all loading points were extracted as the constraints of the following optimization design, and their numerical values are displayed in Table 1 . The total mass of the structure is $7.1 \mathrm{Kg}$. 
Table 1 Stiffness requirements

\begin{tabular}{cccccccc}
\hline & & \multicolumn{5}{c}{ Working conditions } & first natural \\
\cline { 3 - 8 } Displacement(mm) & & Load-1 & Load-2 & Load-3 & Load-4 & Load-5 & $\begin{array}{c}\text { frequency } \\
\text { (HZ) }\end{array}$ \\
\cline { 3 - 8 } & Front-left & $4.010 \mathrm{E}-02$ & $4.052 \mathrm{E}-02$ & $2.006 \mathrm{E}-02$ & $3.355 \mathrm{E}-02$ & $6.459 \mathrm{E}-02$ & \\
\multirow{2}{*}{$\begin{array}{c}\text { Loading } \\
\text { points }\end{array}$} & Mid-left & $1.145 \mathrm{E}-01$ & $1.758 \mathrm{E}-01$ & $6.359 \mathrm{E}-02$ & $1.489 \mathrm{E}-01$ & $1.225 \mathrm{E}-01$ & 501.3 \\
& Rear-left & $1.118 \mathrm{E}-01$ & $1.716 \mathrm{E}-01$ & $4.116 \mathrm{E}-02$ & $1.378 \mathrm{E}-01$ & $7.014 \mathrm{E}-02$ & \\
\hline
\end{tabular}

\section{Mathematical model of shock tower design optimization}

The mathematical model [8] of topology and size optimization of the shock tower can be expressed as:

Minimize: $\quad\left\{\begin{array}{c}\mathrm{W}\left(\rho_{\mathrm{i}}, \mathrm{T}_{\mathrm{j}}\right) \\ \mathrm{F}=\mathrm{KD} \\ \mathrm{f}_{1} \geq \mathrm{F}_{1} \\ \mathrm{~d}_{\mathrm{m}}^{\mathrm{n}} \leq \mathrm{D}_{\mathrm{m}}^{\mathrm{n}} \\ 0<\rho_{\min } \leq \rho_{\mathrm{i}} \leq 1 \\ T_{\mathrm{j}}^{\mathrm{L}} \leq T_{\mathrm{j}} \leq T_{\mathrm{j}}^{U}\end{array}\right.$

Where $\mathrm{W}$ represents the weight of the structure, $\mathrm{K}$ is the global stiffness matrix, $\mathrm{F}$ and $\mathrm{D}$ are the vectors of the nodal force and displacement, respectively, and $f_{1}$ is the first natural frequency, $F_{1}$ is the lower bound for $\mathrm{f}_{1}, \mathrm{~d}_{\mathrm{m}}^{\mathrm{n}}$ denotes the displacement of the $\mathrm{m}$-th loading point in the $\mathrm{n}$-th load condition. $\rho_{i}$ denotes the relative density of the i-th element, $\rho_{\min }$ is the minimum allowable element density, $T_{j}$ represents the $j$-th design variables for size optimization, $T_{j}^{L}$ and $T_{j}^{U}$ are the lower bounds and upper bounds for $\mathrm{T}_{\mathrm{j}}$.

\section{Topology optimization}

The model for topology optimization. The geometric model for topology optimization was established based on original shock tower with an average thickness of $10 \mathrm{~mm}$, and the inner hollow boss structure was filled with solid, as shown in Fig .3(a). Since the topology optimization problem focus on the optimal load transfer path, non-critical region at the junction on boundary was not taken into consideration in the conceptual design stage, and the bolt holes (in blue) were excluded from the domain, which were not involved in optimization. The demoulding constraint (DC) was applied to ensure that there are no obstacles in parting direction. Draft direction represented by red arrow in figure. 3 (a) is perpendicular to boss on the top. Stiffness targets were chosen as the constraint and the objective is to minimize the mass. The material is aluminum alloy.

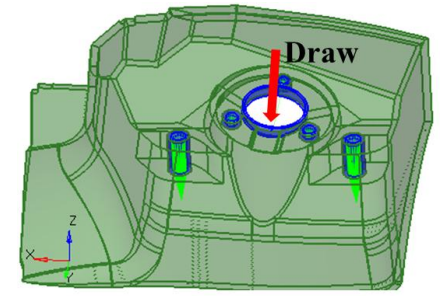

(a)

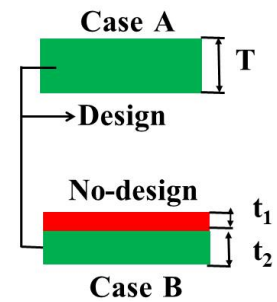

(b)

Fig.3 The geometric model for topology optimization 
In addition, the design of optimization space was taken into account, which is critical and usually neglected. Two cases are given according to the structure and manufacturing processes, as presented in Fig 3(b). It demonstrates the different division of design space in thickness direction, while $\mathrm{t} 1, \mathrm{t} 2$ and $\mathrm{T}$ are respectively $3 \mathrm{~mm}, 7 \mathrm{~mm}$ and $10 \mathrm{~mm}$.

The result of topology optimization. Topology optimization is to find the optimal material distribution satisfying all constraints in the design domain. The results are always characterized by a certain number of intermediate density elements without practical implication. The density contour shown in Fig. 4 indicates the material distribution. Reasonable results can be obtained with a threshold density of 0.3 . Fig. 4(a) is a typical network structure by topology optimization, while Fig. 4(b) is a continuous structure with inner bulky structures. However, both of the optimized structures are far from being ready-to-made. The model was redesigned with casting feasibility in the following step.

The total mass of the structure was decreased with the increased iteration number for the two cases, as is shown in Fig.5. Case B has fewer iteration steps than A for a smaller design space. The final mass is about $3 \mathrm{~kg}$.

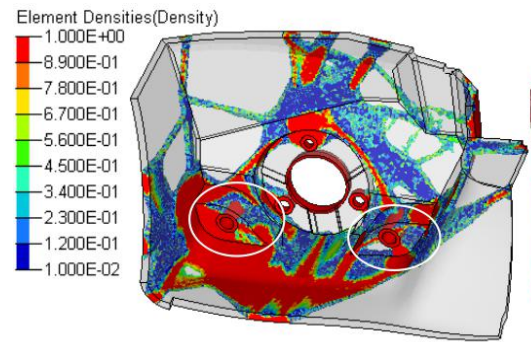

Case A

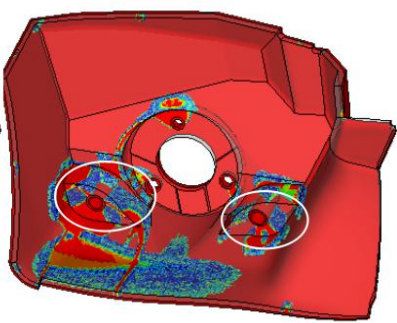

Case B

Fig. 4 The result of topology optimization

\section{Size optimization}

The model for size optimization. To prevent automobile engine compartment from muddy water, the result of case B is preferred in terms of practicability. Since hot spots are easily formed in bulky structures, which results in shrinkage porosity during the casting process, the design domain of case B was interpreted as stiffeners (T2, T3, T4, T6). However, this can lead to insufficient stiffness. Consequently, stiffeners (T1, T5, T7) suggested by the result of case A are added. Since size optimization involves modal analysis in this paper, the model for the size optimization was modeled considering the interface with the adjacent vehicle parts (in yellow).

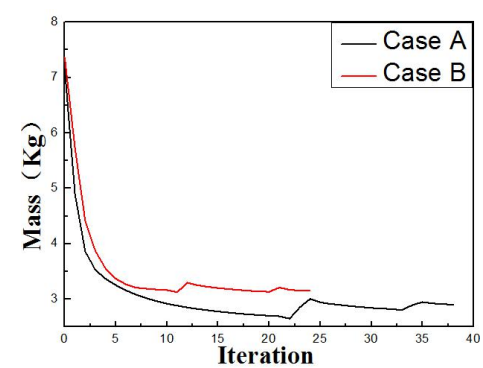

Fig.5 The history of the objective function

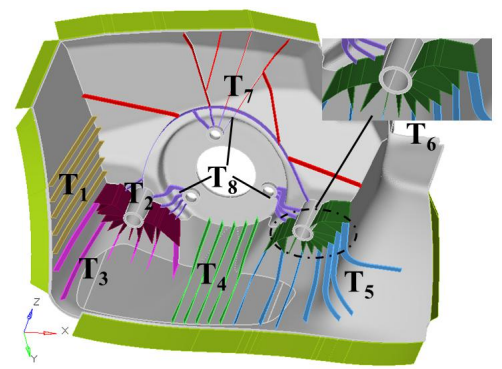

Fig. 6 Geometric model for size optimization

All of the stiffeners were meshed with shell elements for size optimization. The rest of the model was meshed with tetrahedron elements. Constraints and loads were the same as above. In addition, modal constraint that the first natural frequency is no less than $502 \mathrm{~Hz}$ was taken into consideration.

The design variables, exemplified in Fig 6, are distinguished by different colors. The thickness of the design variables were defined as discrete variable with an increment of $0.1 \mathrm{~mm}$. The range of the design variables are listed in Table 2 .

The result of size optimization. The optimal thickness is shown in the last line of table 2 . All of the constraints are satisfied, including 15 stiffness requirements and a natural frequency constraint. The ultimate mass of the structure is $3.9 \mathrm{Kg}$. 
Table 2 Design variables for size optimization

\begin{tabular}{ccccccccc}
\hline Design Variables & T1 & T2 & T3 & T4 & T5 & T6 & T7 & T8 \\
Lower Bound[mm] & 1.0 & 2.0 & 1.0 & 1.0 & 1.0 & 2.0 & 1.0 & 1.0 \\
Upper Bound[mm] & 5.0 & 6.0 & 4.0 & 4.0 & 4.0 & 6.0 & 5.0 & 5.0 \\
Optimal thickness $[\mathrm{mm}]$ & 2.4 & 4.3 & 2.8 & 3.1 & 2.9 & 3.2 & 2.0 & 3.1 \\
\hline
\end{tabular}

\section{Conclusions}

The proposed method was used to solve a multiple loading condition problem concerning structural stiffness and the first natural frequency, which can be potentially extended to shell parts with stiffeners. Topology optimization has shown to be effective and efficient in suggesting an optimal material distribution, while size optimization provides an appropriate thickness for shell elements. The mass of the shock tower has been reduced by nearly $45 \%$ with all the constraints satisfied. Moreover, the division of design space should be addressed, it is also capital that the interpretation of the topology optimization results. Global optimality was not considered in the current paper, additional work is still needed for the validation of the shock resistance and fatigue performance.

\section{Acknowledgements}

This work was financially supported by the Natural Major Research and Development Plan (No. 2016YFB0101603).

\section{References}

[1] Balzer et al. Thin-walled magnesium die-cast shock tower for use in a vehicle, U.S. Patent 9,517,796. (2016)

[2] Dongkai Xu, Jun Chen et al, Topology optimization of die weight reduction for high-strength sheet metal stamping, International Journal of Mechanical Sciences (2012) $73-82$

[3] Sai Zongsheng Wang Guifei et al, Topology and Size Optimization for Landslide Body in NC Lathe Applied Mechanics and Materials (2013) 288: 3-7

[4] Zhao Xinhao, Liu Yanxiong et al, Finite element analysis and topology optimization of a $12000 \mathrm{KN}$ fine blanking press frame, Structural and Multidisciplinary Optimization (2016) $54: 375-389$

[5] Bendsoe MP et al, Optimal shape design as a material distribution problem. Structural Optimization (1989) 1(4):193-202.

[6] Bendsøe MP, Sigmund O, Topology optimization: theory, methods and applications. Springer (2003).

[7] Marco Cavazzuti, Andrea Baldini et al, High performance automotive chassis design: a topology optimization based approach, Structural and Multidisciplinary Optimization (2011) 44:45 - 56

[8] Chao Li, et al, Design space and detailed design of an automotive engineer cradle by using topology, shape, and size optimization. Structural and Multidisciplinary Optimization (2015) 51:547 - 564

[9] Denghong Xiao, Xiandong Liu et al, Application of topology optimization to design an electric bicycle main frame. Structural and Multidisciplinary Optimization (2012) 46:913 - 929

[10] Altair Optistruct (2011) OptiStruct 11.0 User Manual. Altair Engineering, Inc, Troy, MI 